RESPIRATORY INFECTION

\title{
Statin treatment and reduced risk of pneumonia in patients with diabetes
}

\author{
E M W van de Garde, E Hak, P C Souverein, A W Hoes, J M M van den Bosch, \\ H G M Leufkens
}

See end of article for authors' affiliations

\section{Correspondence to:}

E M W van de Garde Utrecht Institute for

Pharmaceutical Sciences

(UIPS), Department of

Pharmacoepidemiology

and Pharmacotherapy,

Sorbonnelaan 16,3583

CA Utrecht, The

Netherlands; e.m.w.

vandegarde@pharm.uu.nl

Received 21 March 2006

Accepted 21 June 2006

Published Online First

29 June 2006
Background: Recent prognostic studies have shown that previous treatment with statins is associated with a better outcome in patients admitted to hospital with pneumonia. Because of an increased risk of pneumonia in patients with diabetes, we assessed the effects of statin use on the occurrence of pneumonia in adult diabetic patients.

Methods: All patients with a diagnosis of diabetes (types 1 and 2) enlisted in the UK General Practice Research Database between 1 June 1987 and 21 January 2001 were included. A case-control study was performed with cases defined as patients with a first recorded diagnosis of pneumonia. For each case up to four controls were matched by age, sex, practice, and index date. Patients were classified as current users when the index date was between the start and end date of statin treatment. Conditional multiple logistic regression analysis was used to estimate the strength of the association between statin treatment and the occurrence of pneumonia.

Results: Statins were used in $1.1 \%$ of 4719 cases and in $2.1 \%$ of 15322 matched controls (crude odds ratio (OR) $0.51,95 \% \mathrm{Cl} 0.37$ to 0.68 ). After adjusting for potential confounders, treatment with statins was associated with a significant reduction in the risk of pneumonia (adjusted OR $0.49,95 \% \mathrm{Cl} 0.35$ to 0.69 ). The association was consistent among relevant subgroups (cardiovascular diseases, pulmonary diseases) and independent of the use of other prescription drugs.

Conclusions: The use of statins is associated with a considerable reduction in the risk of pneumonia in diabetic patients. In addition to lowering the risk of cardiovascular disease, statins may be useful in preventing respiratory infections.
$\mathrm{C}$ ommunity acquired pneumonia ranks in the top 10 leading causes of death in the elderly and is a significant and increasing cause of primary care consultations and hospital admissions. ${ }^{1-3}$ Individuals with co-morbid conditions such as diabetes types 1 and 2 are at increased risk for lower respiratory tract infections and their complications. ${ }^{45}$ Furthermore, the impact of diabetes on deaths associated with pneumonia is substantial. ${ }^{6}$

Recent in vitro studies have shown that inhibitors of hydroxy-methylglutaryl coenzyme A reductase (statins) have different immunomodulatory effects and that statins modulate both lipopolysaccharide induced pulmonary inflammation and pulmonary bacterial infection. ${ }^{7}$ Clinical prognostic studies have confirmed a potential immune regulatory effect and showed that previous treatment with statins was associated with decreased rates of severe sepsis and mortality in patients admitted to hospital with community acquired bacterial infections such as pneumonia. ${ }^{8-12}$ We therefore hypothesised that, if the use of statins can improve the outcome in pneumonia, statin treatment may prevent development of the infection itself. The aim of this study was to assess whether the use of statins is associated with a decreased risk of developing pneumonia in an ambulatory population of diabetic patients.

\section{METHODS}

\section{Data source}

Data were obtained from the United Kingdom General Practice Research Database (GPRD) which contains the computerised medical records of approximately 650 general practices. The GPRD is owned by the UK Department of Health and managed by the Medicines Control Agency.
Approximately $6.5 \%$ of the total population of England and Wales is represented in the database. The computer records contain patient demographic characteristics (height, weight), symptoms and diagnosis (using the Oxford Medical Information System (OXMIS) and Read codes which are mapped onto International Classification of Disease codes), referrals to specialist care, hospital admissions and their major outcomes, and all drug prescriptions in chronological order. The computerised recording of patient information was started by many general practitioners in the late 1980s and replaced the handwritten records used previously. Several independent validation studies have shown that the GPRD database has a high level of completeness and validity. ${ }^{13}{ }^{14}$ The study was approved by the Scientific and Ethical Advisory Group of the GPRD.

\section{Study design and population}

This retrospective case-control study was conducted in a population of 142175 patients with diabetes (both types 1 and 2) recorded in the GPRD from 1 June 1987 to 21 January 2001 (original data extraction early 2002). This population has been used for previous studies on other topics not related to the present research study. ${ }^{15} 16$

Cases were defined as patients aged 18 years and older with a first medical attendance for an episode of community acquired pneumonia (for selected OXMIS and Read codes see Appendix 1). The date of pneumonia diagnosis was recorded as the index date. For each case up to four controls were matched for sex, age ( \pm 2 years), general practice, and index date of the case. Controls were randomly selected from the baseline cohort of diabetic patients without a record of pneumonia. To be able to control for potential prognostic 
differences between comparison groups, both cases and controls were eligible for inclusion in the study if they had a medical history in the database for at least 365 days before the index date.

\section{Exposure to statins}

For each patient we identified all prescriptions for statins before the index date (atorvastatin, cerivastatin, fluvastatin, pravastatin, simvastatin). Episodes of statin treatment were defined as a series of subsequent prescription refills for these drugs. We assumed that a new episode of treatment started if an interval of 14 days or more occurred between the theoretical end date of a prescription and the date of the next prescription for the same patient. The theoretical end date was defined as the prescription date plus the duration of use (number of tablets prescribed divided by the prescribed daily dose). Patients were classified as current statin users when the index date was between the start and the end date of a treatment episode. Patients were classified as past users when they were not a current user but had a history of use in the year before the index date.

\section{Potential confounders}

To be able to control for potential confounding, medical information was obtained on pneumonia risk factors that could potentially confound the association between statin treatment and outcome. For each patient we evaluated the presence or absence of the following frequently occurring comorbidities as potential confounders: cardiovascular diseases (congestive heart failure, stroke), pulmonary diseases, and alcoholism. ${ }^{17-20}$ In addition, the latest data on smoking status (non-smoking, ex-smoking, current smoking, or unknown) and body mass index $\left(<20,20-24,25-30,>30 \mathrm{~kg} / \mathrm{m}^{2}\right.$, or unknown) were assessed. Possible confounding drugs examined were influenza vaccination, pneumococcal vaccination, immunosuppressive drugs (methotrexate, cyclosporin, tacrolimus, etanercept, infliximab, fludarabin, cladrimycin, rituximab, or alemtuzimab), oral glucocorticoids, and use of gastric acid suppressing drugs. ${ }^{21-24} \mathrm{~A}$ patient was considered to have been exposed to a drug if more than one prescription was issued in the year before the index date, except for vaccination drugs which required only one prescription. The prescription drugs shown above were selected because they have been linked to a decreased or increased risk of pneumonia, and those receiving these drugs may be more likely to receive statins because of their likelihood of attending a physician. We calculated the number of general practitioner (GP) visits in the year before the index date as a proxy for overall health status and healthcare consumption.

\section{Statistical analysis}

Univariate analysis by $\chi^{2}$ tests and Student $t$ tests was used to test for statistically significant differences in baseline characteristics between cases and controls. Conditional logistic regression was used to estimate the strength of the association between statin treatment and the risk of pneumonia and expressed as odds ratios (OR) with 95\% confidence intervals (CI). We included all potential confounders and age in the multivariate analysis to adjust for the baseline differences between both groups. Exposure to statins was categorised as current, past, and never (reference). In addition, to elucidate a possible time trend effect, the study population was divided into quartiles based on ranked index dates and analysed separately. Stratified analyses were conducted to detect possible differences in effect between co-morbidity related subgroups.

\section{RESULTS}

The study population comprised 4719 patients with a diagnosis of pneumonia and 15322 matched controls. A diagnosis of pneumococcal pneumonia was recorded for 271 of the cases, 46 had a diagnosis of other bacterial pneumonia,

\begin{tabular}{|c|c|c|c|}
\hline Characteristic & $\begin{array}{l}\text { Cases }(n=4719) \\
n(\%)\end{array}$ & $\begin{array}{l}\text { Controls }(n=15322) \\
n(\%)\end{array}$ & $\begin{array}{l}\text { Crude OR } \\
(95 \% \mathrm{CI})\end{array}$ \\
\hline \multicolumn{4}{|l|}{ Age (years) } \\
\hline$<60$ & 525 (11.1\%) & $1776(11.6 \%)$ & * \\
\hline $60-69$ & $785(16.6 \%)$ & $3066(20.0 \%)$ & * \\
\hline 70-79 & $1533(32.5 \%)$ & $5665(37.0 \%)$ & * \\
\hline 80-89 & $1622(34.4 \%)$ & $4431(28.9 \%)$ & * \\
\hline$\geqslant 90$ & $254(5.4 \%)$ & $384(2.5 \%)$ & * \\
\hline \multicolumn{4}{|l|}{ Sex } \\
\hline Male & $2275(48.2 \%)$ & $7336(47.9 \%)$ & * \\
\hline Female & $2444(51.8 \%)$ & $7986(42.1 \%)$ & * \\
\hline \multicolumn{4}{|l|}{ Co-morbidities } \\
\hline Cardiovascular disease & $2189(46.4 \%)$ & $3611(23.6 \%)$ & 2.81 (2.62 to 3.00$)$ \\
\hline Pulmonary disease & $949(20.1 \%)$ & $1592(10.4 \%)$ & 2.17 (1.99 to 2.37 ) \\
\hline Alcoholism & $17(0.4 \%)$ & $34(0.2 \%)$ & $1.62(0.91$ to 2.91$)$ \\
\hline \multicolumn{4}{|l|}{ Body mass index $\left(\mathrm{kg} / \mathrm{m}^{2}\right)$} \\
\hline $20-24$ & $689(14.6 \%)$ & $2215(14.5 \%)$ & 1.00 (reference) \\
\hline$<20$ & $251(5.3 \%)$ & $466(3.0 \%)$ & $1.73(1.45$ to 2.06$)$ \\
\hline $25-30$ & 1083 (22.9\%) & $4939(32.2 \%)$ & $0.71(0.63$ to 0.79$)$ \\
\hline$>30$ & 485 (10.3\%) & $2365(15.4 \%)$ & 0.66 (0.58 to 0.75$)$ \\
\hline Unknown & 2211 (46.9\%) & $5337(34.8 \%)$ & $1.33(1.21$ to 1.47$)$ \\
\hline \multicolumn{4}{|l|}{ Prescription drugs } \\
\hline Flu vaccination & $1955(41.4 \%)$ & $6114(39.9 \%)$ & 1.07 (1.00 to 1.14$)$ \\
\hline Gastric acid suppressing & $967(20.5 \%)$ & 1988 (13.0\%) & $1.73(1.59$ to 1.88$)$ \\
\hline \multicolumn{4}{|l|}{ drugs } \\
\hline Immunosuppressive drugs & $27(0.6 \%)$ & $38(0.2 \%)$ & $2.32(1.41$ to 3.80$)$ \\
\hline Oral glucocorticoids & $311(6.6 \%)$ & $329(2.1 \%)$ & 3.22 (2.74 to 3.77$)$ \\
\hline Pneumococcal vaccination & $144(3.1 \%)$ & $569(3.7 \%)$ & $0.82(0.68$ to 0.98$)$ \\
\hline Statins (current use) & $50(1.1 \%)$ & $318(2.1 \%)$ & 0.51 (0.37 to 0.68$)$ \\
\hline Mean (SD) no GP visits/year & $14.8(11.0)$ & $9.1(7.7)$ & N/A \\
\hline Current smokingt & 407 (12.7\%) & 1169 (9.2\%) & $1.48(1.31$ to 1.67$)$ \\
\hline $\begin{array}{l}\text { Matching variable. } \\
\text { Percentage for current smo } \\
\text { GPRD }\end{array}$ & 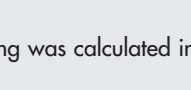 & & 1. \\
\hline
\end{tabular}


Table 2 Association between current statin treatment and the occurrence of pneumonia in all patients and in different (co-morbidity) subgroups

\begin{tabular}{|c|c|c|}
\hline (Sub)groups & $\begin{array}{l}\text { Crude OR } \\
(95 \% \mathrm{Cl})\end{array}$ & $\begin{array}{l}\text { Adjusted* OR } \\
(95 \% \mathrm{CI})\end{array}$ \\
\hline All patients & 0.51 (0.37 to 0.68$)$ & $0.49(0.35$ to 0.69$)$ \\
\hline Men & $0.65(0.42$ to 1.02$)$ & $0.56(0.39$ to 0.91$)$ \\
\hline Women & 0.51 (0.33 to 0.78$)$ & $0.40(0.25$ to 0.62$)$ \\
\hline No prior cardiovascular diseases & $0.63(0.42$ to 0.93$)$ & $0.60(0.40$ to 0.91$)$ \\
\hline Prior cardiovascular diseases & $0.35(0.21$ to 0.57$)$ & $0.36(0.22$ to 0.62$)$ \\
\hline No prior pulmonary diseases & 0.50 (0.35 to 0.72$)$ & $0.41(0.28$ to 0.61$)$ \\
\hline Prior pulmonary diseases & 0.87 (0.47 to 1.58 ) & 0.85 (0.45 to 1.63$)$ \\
\hline \multicolumn{3}{|c|}{$\begin{array}{l}\text { OR, odds ratio; } \mathrm{Cl} \text {, confidence interval. } \\
\text { *Adjusted for age, cardiovascular diseases, pulmonary diseases, smoking, } \\
\text { alcoholism, body mass index, gastric acid suppressing drug, flu vaccination, } \\
\text { immunosuppressive drugs, pneumococcal vaccination, oral glucocorticoids, } \\
\text { and number of GP contacts, with the exception of the grouping variable in the } \\
\text { subgroup analysis. }\end{array}$} \\
\hline
\end{tabular}

2050 had a diagnosis of bronchopneumonia with unspecified organism recorded, and 2291 had pneumonia with unspecified organism recorded. $28 \%$ of cases were referred to hospital because of their pneumonia diagnosis. About $48 \%$ of cases and controls were men; the mean age of both cases and controls was 73 years but the proportion of very old was higher among the cases than in the controls (table 1). Matching on age had a median difference in age of 1.00 year with a mean difference of -0.16 years. The median duration of observation in the GPRD was 4.0 years for cases and 4.1 years for controls.

The presence of cardiovascular diseases, pulmonary diseases, smoking, and alcoholism was higher in cases than in controls, as was the use of gastric acid suppressing drugs and oral glucocorticoids. Cases visited the GP more often than controls ( $15 \vee 9$ visits per year, respectively, $\mathrm{p}<0.01$ ).

Of the 4719 cases, $50(1.1 \%)$ were on active statin treatment compared with $2.1 \%$ for the control group. Univariate analysis showed that current statin treatment was associated with a reduced risk of pneumonia (crude OR 0.51 , 95\% CI 0.37 to 0.68). After adjusting for age, cardiovascular diseases, pulmonary diseases, body mass

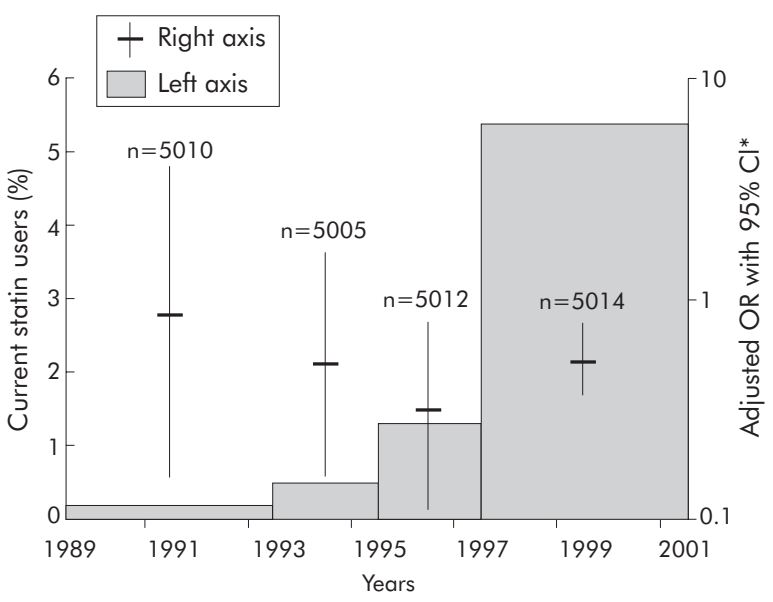

Figure 1 Prevalence of current statin use (left axis) and the association between statin use and the occurrence of pneumonia (right axis) in different time windows based on quartiles of ranked index dates of all subjects. OR, odds ratio; $\mathrm{Cl}$, confidence interval. *Adjusted for age, cardiovascular diseases, pulmonary diseases, smoking, alcoholism, body mass index, gastric acid suppressing drug, flu vaccination, immunosuppressive drugs, pneumococcal vaccination, oral glucocorticoids, and number of GP contacts. $n=$ number of subjects in quartile. index, alcoholism, smoking, influenza vaccination, pneumococcal vaccination, gastric acid suppressing drugs, immunosuppressive drugs, oral glucocorticoids, and number of GP visits in the year before the index date, the association did not alter substantially, yielding an adjusted OR of 0.49 (95\% CI 0.35 to 0.69 ). The protective effect was similar for all statins and was consistent for both summer and winter seasons. The adjusted ORs for the different statins were 0.57 (95\% CI 0.30 to 1.11$)$ for atorvastatin $(\mathrm{n}=57), 0.48$ (95\% CI 0.14 to 1.71$)$ for cerivastatin $(\mathrm{n}=17), 0.37$ (95\% CI 0.10 to $1.31)$ for fluvastatin $(n=19), 0.36(95 \%$ CI 0.17 to 0.75$)$ for pravastatin $(\mathrm{n}=47)$, and $0.52(95 \%$ CI 0.35 to 0.76$)$ for simvastatin $(n=228)$. Comparable effects were observed in all co-morbidity related subgroups with a strongest association in patients with a history of cardiovascular disease (table 2).

The effect did not differ substantially between younger ( $\leqslant 70$ years; OR $0.56,95 \%$ CI 0.37 to 0.86 ) and older patients (>70 years; OR $0.35,95 \%$ CI 0.20 to 0.61 ), and the effect of current statin treatment on the risk of pneumonia was similar for episodes of community acquired pneumonia treated in primary care (OR $0.52,95 \%$ CI 0.36 to 0.77 ) and those requiring admission to hospital (OR 0.50, 95\% CI 0.28 to 0.89 ). Furthermore, the association was equally present in the more recent years of the study period and in the first years, despite a consistent increase in statin prescriptions with time (fig 1). Past use of statins was not associated with a decreased risk of pneumonia (OR $0.95,95 \%$ CI 0.63 to 1.42 ).

\section{DISCUSSION}

In this study of diabetic patients the use of statins was associated with a considerably reduced risk of pneumonia. These findings add to the accumulating evidence that statins may have immunomodulatory and anti-inflammatory properties in addition to their lipid lowering effects. In vitro studies have indicated that statins have a potentially beneficial effect by normalising the inflammatory response in respiratory bacterial infections. ${ }^{925}$ This finding seems to have been confirmed by several prognostic clinical studies which found a substantial reduction in severe outcomes among statin users with pneumonia. ${ }^{78}$ Our large casecontrol study with a long study period and a representative high risk population is the first to extend these findings to examine the possible prophylactic effect of statins on the incidence of pneumonia.

This is important as it suggests that statins not only influence the development of pulmonary infection but could also prevent primary infection. The mechanisms behind this protective effect, however, remain speculative. To our knowledge, the only study addressing the possible effects of statins on primary host defence is the experimental study by Fessler et $a l^{7}$ which suggested impaired host defence in a murine model. However, there are several limitations in that study which preclude translation to the clinical situation. These include (1) the differences between a murine model and men; (2) the very high doses of lovastatin used ( 10 times that routinely used in humans); (3) observation of mice for only 48 hours after intratracheal ingestion of bacteria; and (4) lack of correlation between the number of colony forming units and clinical appearance. Furthermore, it is possible that the other anti-inflammatory effects of statins (on macrophages and monocytes) may outweigh any impaired neutrophilic microbicidal capacity.

The prevalence of statin use in our study was relatively low, certainly in comparison with recent years. This is primarily caused by the period under study. We think, however, that this has no impact on the validity of our study. Firstly, no obvious time dependent effect was observed (fig l). The association was consistently pronounced in the more recent 
years of the study period compared with earlier years, despite a constant increase in the use of statins with time. The large confidence interval in the first quartiles is most likely explained by small numbers. Additionally, although the power to detect differences between individual statins is somewhat limited, all the statins had odds ratios in the same range below 1 . This suggests that the effect of statins results from their intrinsic properties and is independent of trends in the prescribing of statins. Furthermore, adjustment of the associations for the presence of many important and frequently occurring co-morbidities and use of other prescription drugs did not substantially change the risk estimates. However, we cannot rule out the possibility of confounding by unknown or unmeasured factors.

This study was conducted in a high risk population of diabetic patients. Although patients with diabetes are more susceptible to infection, to our knowledge the mechanism of action of statins does not differ between diabetic and nondiabetic patients. The protective properties of statins may therefore be applicable to the general population, but further research is needed to establish a possible protective effect in non-diabetic patients.

Our study has some limitations. Firstly, identification of cases of pneumonia has the potential for misclassification due to incorrect coding. It is possible that, because of the broad definitions used for the diagnosis of community acquired pneumonia, GPs could have applied the diagnostic criteria differently - for example, some acute exacerbations of chronic obstructive pulmonary disease could be identified as pneumonia. We believe, however, that such misclassification is independent of statin use and would only lead to an underestimation of the true association between statins and the occurrence of pneumonia. In addition, the overall validity of the GPRD coding has previously been assessed several times and other validation studies on pneumonia coding in different medical databases have also shown positive predictive values ranging from $61 \%$ to $85 \% .^{26}{ }^{27}$ Assuming that the diagnosis of patients admitted to a hospital is more accurate (better accessibility to chest radiography and sputum and blood cultures), it is reassuring that the association between statin use and pneumonia in admitted hospital was identical to that in patients treated in primary care. In agreement with Laheij et al, ${ }^{24}$ our study also showed an association between the use of gastric acid suppressing drugs and pneumonia.

A second possible limitation is misclassification of exposure to statin treatment since we used prescription data. Patients using statins could have been non-compliant with their treatment and therefore could have used fewer statins than were prescribed. However, this would have led to an underestimation of the association between statin use and the occurrence of pneumonia.

Another concern is that people on statins take more comfort out of good health support-the so-called "healthy user effect"-which can induce confounding. It is possible that statins were preferentially prescribed to patients with higher socioeconomic status and a lower risk of pneumonia. We tried to reduce such confounding by matching subjects on general practice (thereby on geographical region and city area). However, the use of postcodes as a proxy for socioeconomic status has been debated in the literature. ${ }^{28} 29$ On the other hand, greater co-morbidity (and therefore a greater risk of pneumonia) could decrease the likelihood that an older person will receive effective treatment for asymptomatic conditions such as raised cholesterol levels. If this was the case, a substantial difference would have been seen between younger and older patients in the protective association between statins and pneumonia and this was not seen in our study.
Finally, we cannot rule out the possibility of confounding by unmeasured factors. However, the finding that the protective effect disappeared after discontinuing statin treatment strongly suggests that it is attributable to the properties of statins.

It must be emphasised that these findings on the preventive potential of statins should not be interpreted as suggesting that statins should be given to all diabetic patients to prevent pneumonia. Further confirmation is needed, particularly from large scale prospective randomised trials. In influenza pandemics statins could possibly provide additional support in the prevention of bacterial superinfections of the lung.

In conclusion, treatment with statins is associated with a considerably decreased risk of pneumonia in diabetic patients and their effects were consistent in all clinically relevant subgroups studied. Evidence is accumulating that treatment with statins may also influence bacterial respiratory infections, which may therefore broaden the indications for these drugs.

\section{Authors' affiliations}

\section{E M W van de Garde, P C Souverein, H G M Leufkens, Utrecht Institute} for Pharmaceutical Sciences (UIPS), Department of

Pharmacoepidemiology and Pharmacotherapy, Utrecht, The Netherlands

E M W van de Garde, St Antonius Hospital, Department of Clinical Pharmacy, Nieuwegein, The Netherlands

E Hak, A W Hoes, University Medical Center Utrecht, Julius Center for Health Sciences and Primary Care, Utrecht, The Netherlands

J M M van den Bosch, St Antonius Hospital, Department of Pulmonary Diseases, Nieuwegein, The Netherlands

None of the authors is involved in any organisation or entity with a financial interest in or financial conflict with the subject matter or materials discussed in the manuscript.

None of the authors has in the past five years accepted the following from an organisation that may in any way gain or lose financially from the results of the study: reimbursement for attending a symposium, a fee for speaking, a fee for organising education, funds for research, funds for a member of staff, fees for consulting. None of the authors has in the past five years been employed by an organisation that may in any way gain or lose financially from the results or conclusions of the study, or holds any stocks or shares in an organisation that may in any way gain or lose financially from the results or conclusions of the study.

\begin{tabular}{|ll|}
\hline \multicolumn{2}{|l|}{$\begin{array}{l}\text { Appendix } 1 \text { Medical codes used for case } \\
\text { selection }\end{array}$} \\
\hline Code & Description \\
\hline H28..00 & Atypical pneumonia \\
H261.0 & Basal pneumonia due to unspecified organism \\
485 & Bronchopneumonia \\
H25..00 & Bronchopneumonia due to unspecified organism \\
H21..00 & Lobar (pneumococcal) pneumonia \\
481 B & Lobar pneumonia \\
H260.0 & Lobar pneumonia due to unspecified organism \\
H22..00 & Other bacterial pneumonia \\
486 & Pneumonia \\
H2...0 & Pneumonia and influenza \\
486 AP & Pneumonia aspiration \\
483 AT & Pneumonia atypical \\
481 BA & Pneumonia basal \\
H26..00 & Pneumonia due to unspecified organism \\
$483 \mathrm{M}$ & Pneumonia mycoplasal \\
H2z..00 & Pneumonia or influenza nos \\
$481 \mathrm{~A}$ & Pneumonia pneumococcal \\
4823 & Pneumonia staphylococcal \\
$486 \mathrm{~T}$ & Pneumonitis \\
\hline & \\
\hline
\end{tabular}




\section{REFERENCES}

1 Mortensen EM, Coley CM, Singer DE, et al. Causes of death for patients with community-acquired pneumonia: results from the Pneumonia Patient Outcomes Research Team cohort study. Arch Intern Med 2002; 162:1059-64.

2 Fine MJ, Smith MA, Carson CA, et al. Prognosis and outcomes of patients with community-acquired pneumonia. A meta-analysis. JAMA 1996;275:134-41.

3 Fry AM, Shay DK, Holman RC, et al. Trends in hospitalizations for pneumonia among persons aged 65 years or older in the United States, 1988-2002. JAMA 2005;294:2712-9.

4 Moutschen $M$. Alterations in natural immunity and risk of infection in patients with diabetes mellitus (in Belgian). Rev Med Liege 2005;60:541-4.

5 Muller LM, Gorter KJ, Hak E, et al. Increased risk of common infections in patients with type 1 and type 2 diabetes mellitus. Clin Infect Dis 2005;41:281-8.

6 Valdez R, Narayan KM, Geiss LS, et al. Impact of diabetes mellitus on mortality associated with pneumonia and influenza among non-Hispanic black and white US adults. Am J Public Health 1999;89:1715-21.

7 Fessler MB, Young SK, Jeyaseelan S, et al. A role for hydroxy-methylglutaryl coenzyme a reductase in pulmonary inflammation and host defense. Am J Respir Crit Care Med 2005;171:606-15.

8 Almog Y, Shefer A, Novack V, et al. Prior statin therapy is associated with a decreased rate of severe sepsis. Circulation 2004;110:880-5.

9 Almog Y. Statins, inflammation, and sepsis: hypothesis. Chest 2003;124:740-3

10 Mortensen EM, Restrepo MI, Anzueto A, et al. The effect of prior statin use on 30-day mortality for patients hospitalized with community-acquired pneumonia. Respir Res 2005;6:82.

11 Hackam DG, Mamdani M, Li P, et al. Statins and sepsis in patients with cardiovascular disease: a population-based cohort analysis. Lancet 2006;367:413-8.

12 Thomsen RW, Hundborg HH, Johnsen SP, et al. Statin use and mortality within 180 days after bacteremia: a population-based cohort study. Crit Care Med 2006;34:1080-6

13 Jick H, Jick SS, Derby LE. Validation of information recorded on general practitioner based computerised data resource in the United Kingdom. BMJ $1991 ; 302: 766-8$.

14 Jick SS, Kaye JA, Vasilakis-Scaramozza C, et al. Validity of the general practice research database. Pharmacotherapy 2003;23:686-9.
15 De Bruin ML, van Staa TP, Belitser SV, et al. Predicting cardiac arrhythmias and sudden death in diabetic users of proarrhythmic drugs. Diabetes Care 2005;28:440-2.

16 Verdel BM, Souverein PC, Egberts AC, et al. Difference in risks of allergic reaction to sulfonamide drugs based on chemical structure. Ann Pharmacother 2006;40:1040-6.

17 Koivula I, Sten M, Makela PH. Risk factors for pneumonia in the elderly. Am J Med 1994;96:313-20.

18 Almirall J, Bolibar I, Balanzo X, et al. Risk factors for community-acquired pneumonia in adults: a population-based case-control study. Eur Respir J 1999; 13:349-55.

19 Ruiz M, Ewig S, Marcos MA, et al. Etiology of community-acquired pneumonia: impact of age, comorbidity, and severity. Am J Respir Crit Care Med 1999;160:397-405.

20 Nakagawa T, Sekizawa K, Arai $\mathrm{H}$, et al. High incidence of pneumonia in elderly patients with basal ganglia infarction. Arch Intern Med 1997; 157:321-4.

21 White DA. Drug-induced pulmonary infection. Clin Chest Med 2004;25: 179-87.

22 Jefferson T, Rivetti D, Rivetti A, et al. Efficacy and effectiveness of influenza vaccines in elderly people: a systematic review. Lancet 2005;366:1165-74.

23 Shorr AF. Preventing pneumonia: the role for pneumococcal and influenza vaccines. Clin Chest Med 2005;26:123-34.

24 Laheij RJ, Sturkenboom MC, Hassing RJ, et al. Risk of community-acquired pneumonia and use of gastric acid-suppressive drugs. JAMA 2004;292:1955-60.

25 Kwak B, Mulhaupt F, Myit S, et al. Statins as a newly recognized type of immunomodulator. Nat Med 2000;6:1399-402.

26 Guevara RE, Butler JC, Marston BJ, et al. Accuracy of ICD-9-CM codes in detecting community-acquired pneumococcal pneumonia for incidence and vaccine efficacy studies. Am J Epidemiol 1999;149:282-9.

27 Aronsky D, Haug PJ, Lagor C, et al. Accuracy of administrative data for identifying patients with pneumonia, Am J Med Qual 2005;20:319-28.

28 McLoone P, Ellaway A. Postcodes don't indicate individuals' social class. BMJ 1999;319:1003-4.

29 Danesh J, Gault S, Semmence J, et al. Postcodes as useful markers of social class: population based study in 26000 British households. BMJ 1999;318:843-4.

\section{LUNG ALERT}

No further tests for PE required in low probability cases with a negative D-dimer test

$\Delta$ Kearon C, Ginsberg JS, Douketis J, et al for the Canadian Pulmonary Embolism Study Group. An evaluation of D-dimer in the diagnosis of pulmonary embolism. Ann Intern Med 2006;144:812-21

$\mathrm{n}$ patients with suspected pulmonary embolism (PE), further diagnostic testing is often omitted on the basis of a negative D-dimer test result. This study is the first randomised controlled trial to evaluate this approach.

1126 randomised patients with suspected PE were evaluated and classified into low (group A) and high (group B) clinical probability groups; 456 with negative D-dimer test results were randomly allocated to control or experimental interventions. The experimental intervention for both groups was no further testing. The control intervention for group A was a ventilation-perfusion (VQ) scan followed by bilateral leg proximal vein ultrasonography. If the VQ scan was equivocal, ultrasonography was repeated at 7 and 14 days. The control intervention for group B was ultrasonography at 7 and 14 days. All patients in group B had a baseline VQ scan.

The patients were followed up for symptomatic venous thromboembolism (VTE) over a 6 month period. The prevalence of VTE in the 1126 patients enrolled in the study was $15.2 \%$. In group A, six patients were lost to follow up and VTE occurred in 0 of 182 patients in the experimental group and 1 of 185 in the control group (95\% CI -3 to 1.6 percentage points). In group B, VTE occurred in 1 of 41 patients in the experimental group and 0 of 41 in the control group.

The authors conclude that, in patients with a low probability of PE and a negative D-dimer test, additional diagnostic testing can be withheld without increasing the frequency of VTE during follow up. However, the study was limited by its use of VQ scanning and lack of double blinding. Computed tomographic pulmonary angiography (CTPA) is now a more widely used diagnostic test for PE.

N Bari

Specialist Registrar in Respiratory Medicine, Royal London Hospital, London, UK; nabeelab@hotmail.com 